OPEN ACCESS

Edited by:

Vinícius Rosa Cota, Universidade Federal de São João del-Rei, Brazil

Reviewed by: Marta Parazzini, National Research Council (CNR), Italy Pratik Yashvant Chhatbar, Duke University, United States

*Correspondence: Myles Mc Laughlin myles.mclaughlin@kuleuven.be

Specialty section: This article was submitted to Neuroprosthetics, a section of the journal

Frontiers in Neuroscience

Received: 18 September 2021 Accepted: 29 November 2021 Published: 16 December 2021

Citation:

Khatoun A, Asamoah B and Mc Laughlin M (2021) A Computational Modeling Study to Investigate the Use of Epicrania Electrodes to Deliver Interferential Stimulation to Subcortical Regions.

Front. Neurosci. 15:779271 doi: 10.3389/fnins.2021.779271

\section{A Computational Modeling Study to Investigate the Use of Epicranial Electrodes to Deliver Interferential Stimulation to Subcortical Regions}

\author{
Ahmad Khatoun ${ }^{1,2}$, Boateng Asamoah ${ }^{1,2}$ and Myles Mc Laughlin ${ }^{1,2 *}$ \\ 'ExpORL, Department of Neurosciences, KU Leuven, Leuven, Belgium, ${ }^{2}$ The Leuven Brain Institute, KU Leuven, Leuven, \\ Belgium
}

Background: Epicranial cortical stimulation (ECS) is a minimally invasive neuromodulation technique that works by passing electric current between subcutaneous electrodes positioned on the skull. ECS causes a stronger and more focused electric field in the cortex compared to transcranial electric stimulation (TES) where the electrodes are placed on the scalp. However, it is unknown if ECS can target deeper regions where the electric fields become relatively weak and broad. Recently, interferential stimulation (IF) using scalp electrodes has been proposed as a novel technique to target subcortical regions. During IF, two high, but slightly different, frequencies are applied which sum to generate a low frequency field (i.e., $10 \mathrm{~Hz}$ ) at a target subcortical region. We hypothesized that IF using ECS electrodes would cause stronger and more focused subcortical stimulation than that using TES electrodes.

Objective: Use computational modeling to determine if interferential stimulationepicranial cortical stimulation (IF-ECS) can target subcortical regions. Then, compare the focality and field strength of IF-ECS to that of interferential Stimulation-transcranial electric stimulation (IF-TES) in the same subcortical region.

Methods: A human head computational model was developed with 19 TES and 19 ECS disk electrodes positioned on a 10-20 system. After tetrahedral mesh generation the model was imported to $\mathrm{COMSOL}$ where the electric field distribution was calculated for each electrode separately. Then in MATLAB, subcortical targets were defined and the optimal configurations were calculated for both the TES and ECS electrodes.

Results: Interferential stimulation using ECS electrodes can deliver stronger and more focused electric fields to subcortical regions than IF using TES electrodes.

Conclusion: Interferential stimulation combined with ECS is a promising approach for delivering subcortical stimulation without the need for a craniotomy.

Keywords: epicranial electrode, interferential stimulation, subcortical stimulation, transcranial stimulation, neuromodulation 


\section{INTRODUCTION}

The use of electrical brain stimulation to modulate neural activity is a rapidly growing field with many novel methods currently being proposed and evaluated (Lewis et al., 2016; Brittain and Cagnan, 2018; Vöröslakos et al., 2018). One of the more established methods is deep brain stimulation (DBS) which is currently used in the clinic to successfully treat a range of neurological and psychiatric disorders (Lee et al., 2019; Lozano et al., 2019). However, DBS requires a highly invasive neurosurgical procedure to chronically implant electrode arrays in deep brain regions. This increases the invasiveness and brings risks associated with the surgical procedure (Fenoy and Simpson, 2014). Thus, non-invasive electric neuromodulation methods such as transcranial electric stimulation (TES, e.g., transcranial direct current stimulation, transcranial alternating current stimulation, and transcranial random noise stimulation), where electrodes are placed on the scalp, are currently under investigation as non-invasive alternatives (Moreno-Duarte et al., 2014; Bestmann and Walsh, 2017). The use of scalp electrodes means that TES is completely noninvasive. However, this comes at the cost of the electric field strength and focality in the brain because the skin shunts most of the applied current resulting in a weak electric field in the brain. Furthermore, co-stimulation of peripheral nerves in the scalp means that TES becomes painful if amplitudes are increased above $2 \mathrm{~mA}$ (Bikson et al., 2016; Antal et al., 2017). In a recent study, we introduced a novel technique, epicranial cortical stimulation (ECS), and demonstrated its potential at overcoming some of the limitation of TES (Khatoun et al., 2019). In Our computational modeling and animal studies ECS induced stronger and more focused electric fields in the cortex than TES (Khatoun et al., 2019). Yet, ECS requires a minimally invasive surgical intervention to place stimulation electrodes under the scalp and directly over the skull. There is currently an ongoing clinical trial using ECS electrode in epilepsy patients (EASEE, 2021). However, it is currently not known if ECS can also be used to target subcortical regions.

Recently a groundbreaking study introduced a novel electrical brain stimulation technique that can target deep brain regions non-invasively using interfering fields (Grossman et al., 2017). This technique, interferential current stimulation (IF), utilizes the concept of wave interference between two sinusoidal stimulation sources of high but slightly different frequencies (e.g., 5,000 and $5,010 \mathrm{~Hz}$ ) applied to two pairs of TES electrodes. The high frequency fields sum to generate, at a target subcortical region, a low frequency field equivalent to the difference between the two applied high frequencies (i.e., $10 \mathrm{~Hz}$ ). The study used both computational and animal experiments to support the feasibility of this approach.

In a recent computational study on IF, Huang et al. (2020) used an array of TES electrodes for each stimulation source instead of only a pair of electrodes. This provides more flexibility to steer the electric field in the brain. In addition, they developed an algorithm to optimize the electrodes configuration to target a pre-defined subcortical region while avoiding others. Their results show a trade-off between electric field strength and focality. In addition, they demonstrated that the resultant electric field strength at any point had a maximal value equal to the lower electric field strength between the two sources at that specific point. In other words, the resultant low frequency field strength at the predefined target was limited to the strength of each of the two high frequency fields independently. Thus, an approach to increase the electric field strength of both sources in the brain would be valuable.

In this study, we hypothesized that IF using ECS electrodes would produce a stronger and more focused subcortical stimulation than IF using TES electrodes. To test this hypothesis, we used a human head computational model with either a set of 19 ECS electrodes or 19 TES electrodes. Then, we pre-defined a number of subcortical targets and calculated the optimized electrode configurations for each of the two approaches. Finally, we compared the fields' strength and focality obtained with IF-ECS and IF-TES at the target points and showed that IF-ECS can target subcortical regions with more focused and stronger fields than is achievable with IF-TES.

\section{MATERIALS AND METHODS}

\section{Head Computational Model and Electric Field Calculation for Individual Electrodes}

A high-precision human head computational model was generated using the multimodal imaging-based detailed anatomical (MIDA) model (Iacono et al., 2015) and then imported into ScanIP (Simpleware Ltd., Exeter, United Kingdom). The model consists of 115 head tissues and brain structures. These tissues may differ in their electrical conductivity values. However, only a limited number of them has been investigated and validated. Thus, it becomes necessary to simplify the model to a limited number of tissues with known and validated conductivity values. In this study, we followed a similar approach to the majority of the similar modeling studies by combining the different sub-tissues to form the five most essential and commonly used tissues: skin, skull, cerebrospinal fluid (CSF), gray matter (GM), and white matter (WM). This approach was validated by comparing modeling results to intracranial brain recording in humans and non-human primates and was shown to have similar results (Opitz et al., 2016; Huang et al., 2017; Lafon et al., 2017). Arrays of 19 TES or ECS disk (15 mm diameter) electrodes were added based on the 10-20 system. The location of the electrodes were calculated using the "Mesh2EEG" Matlab toolbox (Giacometti et al., 2014). The ECS electrodes were modeled with an insulating back layer that covers them and prevents direct contact with the skin (Khatoun et al., 2019). The electrode contacts were modeled as platinum disks and the insulating back layers as a polydimethylsiloxane (PDMS) silicon material, commonly used for invasive electrodes (Meacham et al., 2008; Guo et al., 2013; 
Ochoa et al., 2013). The insulating back layer had a $20 \mathrm{~mm}$ diameter and $1.6 \mathrm{~mm}$ thickness.

After tetrahedral meshes were generated using ScanIP, the volumetric model was imported to COMSOL multiphysics 5.3 (COMSOL, Inc., Burlington, MA, United States) to calculate the electric field distribution for each electrode separately. Thus, a total of 38 electric field simulations were required. Each simulation estimated the electric field distribution through one of the ECS or TES electrodes with a $1 \mathrm{~mA}$ (zero to peak amplitude) current being set as a boundary condition for the finite element problem. Electric current was applied between the selected electrode and a reference electrode defined as the bottom of the head representing a far body reference electrode (Khatoun et al., 2019). Finally, assuming a quasi-static approximation of Maxwell's equations (valid for frequencies $<1 \mathrm{MHz}$ ), Laplace's equation was solved to estimate the electric field vector (E) at each element in the model:

$$
\begin{aligned}
& \nabla \cdot \sigma \nabla \varphi=0 \\
& \mathrm{E}=\nabla \varphi
\end{aligned}
$$

where $\varphi$ represents the electrical potential vector $\mathrm{E}$ and the electric field vector. The electrical conductivities $(\sigma)$ for the tissues were always: $\sigma_{\text {Skin }}=0.465 \mathrm{~S} / \mathrm{m}, \sigma_{\text {Skull }}=0.01 \mathrm{~S} / \mathrm{m}$, $\sigma_{\mathrm{CSF}}=1.65 \mathrm{~S} / \mathrm{m}, \sigma_{\mathrm{GM}}=0.27 \mathrm{~S} / \mathrm{m}, \sigma_{\mathrm{WM}}=0.126 \mathrm{~S} / \mathrm{m}$, $\sigma_{\mathrm{PDMS}}=10^{-19} \mathrm{~S} / \mathrm{m}, \sigma_{\mathrm{pt}}=9.43 \times 10^{6} \mathrm{~S} / \mathrm{m}$ (Mark, 1999; Peters et al., 2001; Akhtari et al., 2002; Datta et al., 2009; Gabriel et al., 2009; Khatoun et al., 2019). The results for each of the 38 simulations were exported to Matlab (MathWorks, MA, United States) where the optimization method was applied.

\section{Resultant Electric Field During Multi-Electrode Stimulation}

Above, the electric field vector was calculated for each electrode separately assuming that the current is only passed between the selected electrode and the reference. Here, the resultant electric field for simultaneous multiple electrodes stimulation is calculated. Using Matlab, the electric field distributions for ECS and TES electrodes were sorted in separate matrices $A_{N} \times m$ where $m$ represents the number of electrodes and $\mathrm{N}$ the number of brain elements ( $\mathrm{n}$ ) multiplied by $3(N=n \times 3)$ taking into account the electric field at the different coordinate axes (i.e., $x, y$, and $z$ ). Given the matrix A we can now estimate the total absolute electric field (E) generated in the brain by the electrode arrays as following:

$$
\begin{aligned}
& \mathrm{E}=\text { A.s } \\
& \text { Such that } \sum_{i=1}^{m} s_{i}=0 \\
& \text { And } \sum_{i=1}^{m}\left|s_{i}\right| \leq \mathrm{I}_{\max }
\end{aligned}
$$

where $\mathrm{s}$ is a vector of length $\mathrm{m}$ representing the applied electric current to each electrode. Eq. 4 ensures all currents entering the brain must exit and Eq. 5 ensures that the maximum applied electric current is below a maximum value $\mathrm{I}_{\max }$. Therefore, by adjusting the values in vector $\mathrm{s}$, the induced electric field in the brain can be steered. This is essential when aiming to stimulate with a maximum field a given brain area while avoiding others. To maximize the electric field at a target brain element, a similar approach to Huang et al. (2020) was used:

$$
\underset{s}{\operatorname{argmax} e A s}
$$

where $\mathrm{e}$ is the desired brain electric field vector having length $\mathrm{N}$ and specifying which brain element to target and what the desired field direction (i.e., $x, y$, and $z$ ) is. Each element in the vector e has a value between zero and one with higher values indicating the target area. In this study, the target point was set to have a value of one and then to decrease in a Gaussian distance relation when moving away from that target. To minimize the electric field at non-target brain elements the following constraint on the non-target electric field power was applied to the optimization approach (6):

$$
\|\Gamma A s\|^{2} \leq \mathrm{P}_{\max }
$$

such that $(|| .||)$ is the L2-norm and $\Gamma$ is a diagonal matrix with size $\mathrm{N}$ and having diagonal component with values between zero and one to specify the weight of each element in the penalty form. In contrast to e, $\Gamma$ has a value of zero at the target and increases in a Gaussian distance relation when moving away from that target. $\mathrm{P}_{\max }$ is an input parameter that specifies the maximum allowed electric field power in the non-target brain elements. A low $\mathrm{P}_{\max }$ value restricts the electric field in the non-target region but might also limit the applied electric current leading to lower fields in the target. The default value of $\mathrm{P}_{\max }$ was calculated as $\mathrm{P}_{\max 0}=\mathrm{eT} \mathrm{A}\left(\mathrm{A}^{\mathrm{T}} \Gamma^{2} \mathrm{~A}\right)^{-1} \mathrm{~A}^{\mathrm{T}} \mathrm{e}$ (Fernández-Corazza et al., 2020; Huang et al., 2020).

\section{Interferential Stimulation and Electrodes Configuration Optimization}

So far it was assumed that the electric current applied to all electrodes was sinusoidal and from the same source, meaning that they have the same frequency and phase. However, when two groups of electrodes receive electric current from two different sinusoidal sources with slightly different high frequencies, interferential phenomenon occurs. This section aims to calculate the optimal electrode configuration, of both IFECS and IF-TES, to target any pre-selected point in the brain with the resultant interferential low frequency field. Thus, we sought to use an approach similar to Huang et al. (2020). Assuming s1 and s2 are the electric current vectors of the first and the second sinusoidal current sources, respectively, the main objective becomes to optimize them. At any given brain element, the resultant interferential electric field is equal to $2 \times \min \left(|| E_{1}||,|| E_{2}||\right)$ where $E_{1}$ and $E_{2}$ are the electric fields generated by the sources $s_{1}$ and $s_{2}$ at the given brain element, respectively. To maximize the resultant interferential field at 
the target, s1 and s2 were calculated by solving the following optimization problem:

$$
\underset{s 1, s 2}{\operatorname{argmax}} 2 \min \left(|| e^{T} A s 1\|,\| e^{T} A s 2 \|\right)
$$

Such that $\sum_{i=1}^{m} s_{1 i}=\sum_{i=1}^{m} s_{2 i}=0$

$$
\text { And } \begin{aligned}
\sum_{i=1}^{m}\left|s_{1 i}\right| & \leq \mathrm{I}_{\max }, \\
\sum_{i=1}^{m}\left|s_{2 i}\right| & \leq \mathrm{I}_{\max }
\end{aligned}
$$

To minimize the resultant interferential field at the non-target elements the following constraint to the above optimization was applied:

$$
\left\|2 \times \min \left(\left\|e^{T} A s_{1}\right\|,\left\|e^{T} A s_{2}\right\|\right)\right\|^{2} \leq P_{\max }
$$

\section{Targets Preselection and Comparison}

\section{Parameters}

To determine if IF-ECS can target subcortical regions and compare the focality and field strength of IF-ECS to that of IF-TES, a number of subcortical targets were preselected. The selected regions are the left subthalamic nucleus (STN), right
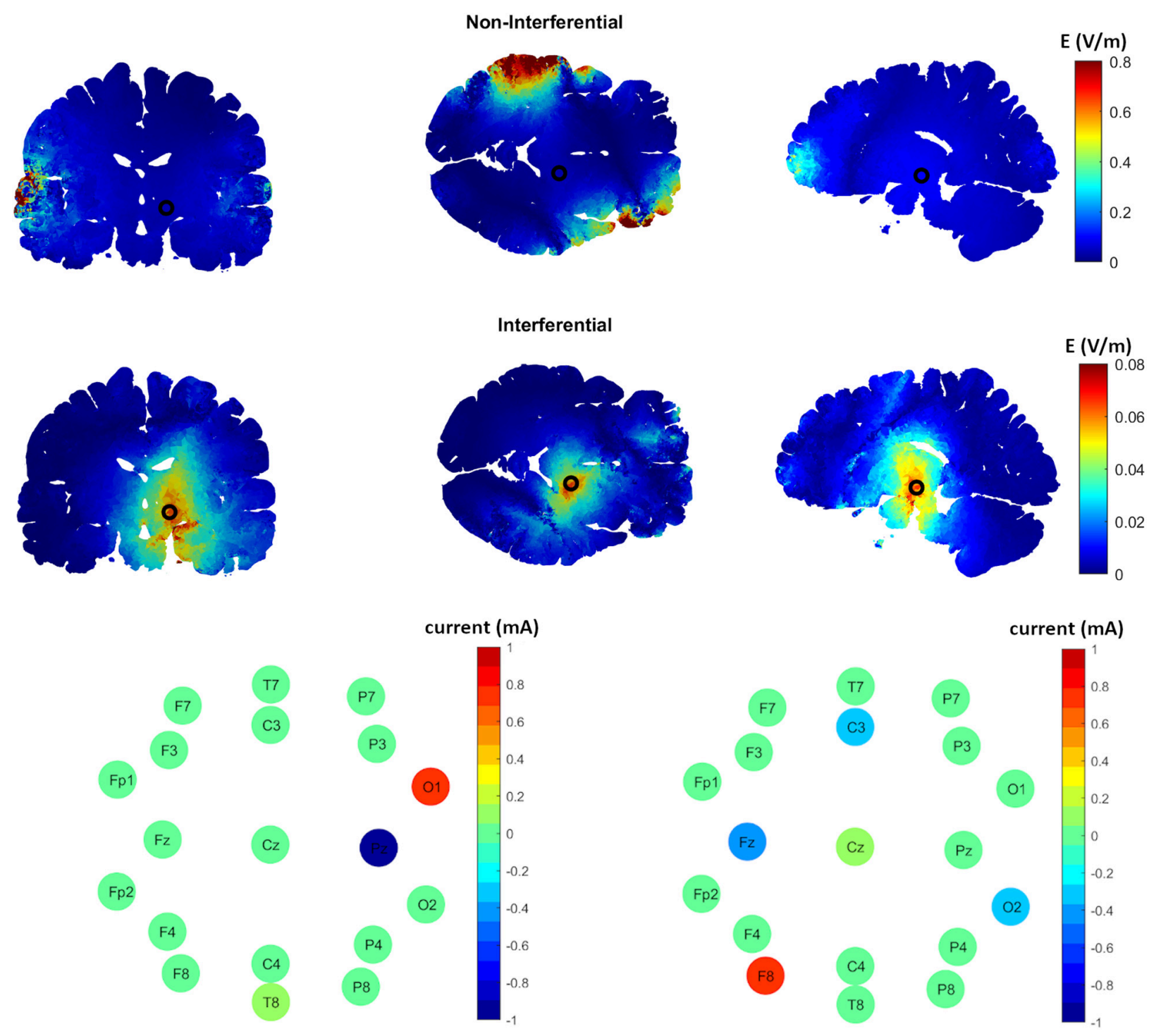

FIGURE 1 | The distribution of the induced interferential and non-interferential electric fields and their corresponding electrodes configuration to target the subthalamic nucleus (STN) using epicranial electrodes. The first row shows the high frequency non-interferential electric field distribution along different brain sections (from left to right, coronal, axial, sagittal). As shown, the highest electric field values are on the cortex under the stimulation electrodes with much weaker fields at the center of the STN (indicated by black circle). On the other hand, the interferential fields (row two) show the strongest values at the target and weaker ones at non-target regions. The third row displays the corresponding optimal electric current distribution along the different electrodes for each of the two sources 1 (left panel) and s2 (right panel). 
ventral intermediate nucleus (VIM), left hypothalamus and the left insula, which are of clinical and research interest for neuromodulation. Each region was defined by one point. Then the optimal electrode configurations were calculated for both IF-ECS and IF-TES for a series of $\mathrm{P}_{\max }$ values as described by Huang et al. (2020). Finally, the electric field strength and focality at the target points were compared between IF-ECS and IFTES. To compare the focality, the half-value volume (F) was calculated (Deng et al., 2013; Khatoun et al., 2018, 2019). This is equivalent to the cubic root of the brain volume having an electric field magnitude higher than half of the electric field strength at the target.

\section{Limiting the Number of Epicranial Electrodes}

Because IF-ECS works using minimally invasive electrodes, limiting the number of implanted electrodes becomes important. However, this comes at the cost of electric field strength and focality. To test the effect of limiting the electrodes number

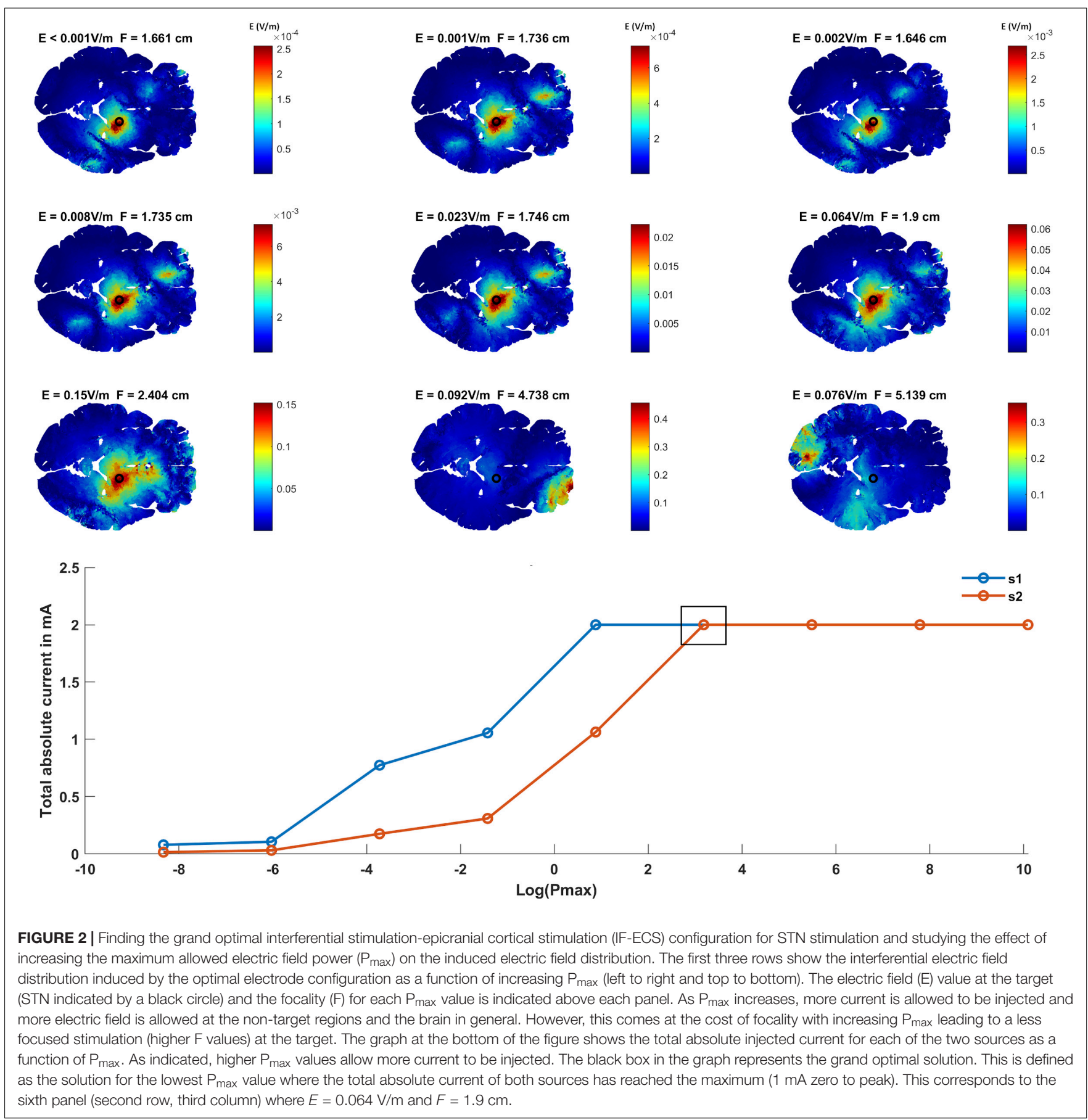


on such parameters, the values were recalculated with only the electrodes with the highest applied current accounting for at least $75 \%$ of the total applied current. Then the applied currents were proportionally readjusted to ensure a total of $1 \mathrm{~mA}$ current was injected. The selection was done for each of the two sources (s1 and s2) and for the cathode and anode poles separately.

\section{RESULTS}

For each of the pre-selected targets the optimal electrode configuration was calculated given a predefined desired electric field direction ( $x$-axis). To adjust the balance between maximizing the interferential field at the target and minimizing the field at the non-target regions, the solution for Eqs. 9-12 was solved for several values of $\mathrm{P}_{\max }$ varying between $\mathrm{P}_{\max 0} \times 10^{-3}$ and $\mathrm{P}_{\max 0} \times 10^{5}$ in a step of $10^{1}$.

\section{Subcortical Brain Stimulation Using Interferential Stimulation-Epicranial Cortical Stimulation}

Figure 1 shows an example of the results obtained using epicranial electrodes. The cross-sectional planes show a relatively high interferential electric field in the target region (STN, with the center indicated by a black circle) and a relatively low interferential electric field in the non-target region (second row, Figure 1). This is not the case for the non-interferential fields where the electric field is highest in the regions under the electrodes (first row, Figure 1); as was shown before (Vöröslakos et al., 2018; Alekseichuk et al., 2019). The electrode configuration for the corresponding results is shown in the third row of Figure 1. The pattern shows that most of the current is applied between electrodes $\mathrm{O} 1$ and $\mathrm{Pz}$ for the first source s1, and F8, Fz, $\mathrm{C} 3$, and $\mathrm{O} 2$ for the source s2.

\section{A Tradeoff Between Field Strength and Focality}

The optimization method aims to maximize the interferential fields at the target and minimize it elsewhere. To adjust the leverage between the two factors, $P_{\max }$ was varied. Figure 2 shows the optimized interferential fields as a function of $\mathrm{P}_{\max }$. Low $\mathrm{P}_{\max }$ values show a more focused but weaker interferential field at the target. As $\mathrm{P}_{\max }$ increases, the focality decreases and the strength of the field at the target increases. This is because as $\mathrm{P}_{\max }$ increases, more electric fields are allowed in the non-target regions and thus more current can be injected. The graph at the bottom of Figure 2 shows the applied current amplitudes of each source s1 and $s 2$ as a function of $P_{\max }$. The small square box indicates the grand optimal solution which represents the solution for the lowest $\mathrm{P}_{\max }$ value where the total current applied to each of the two high frequency sources has reached maximum

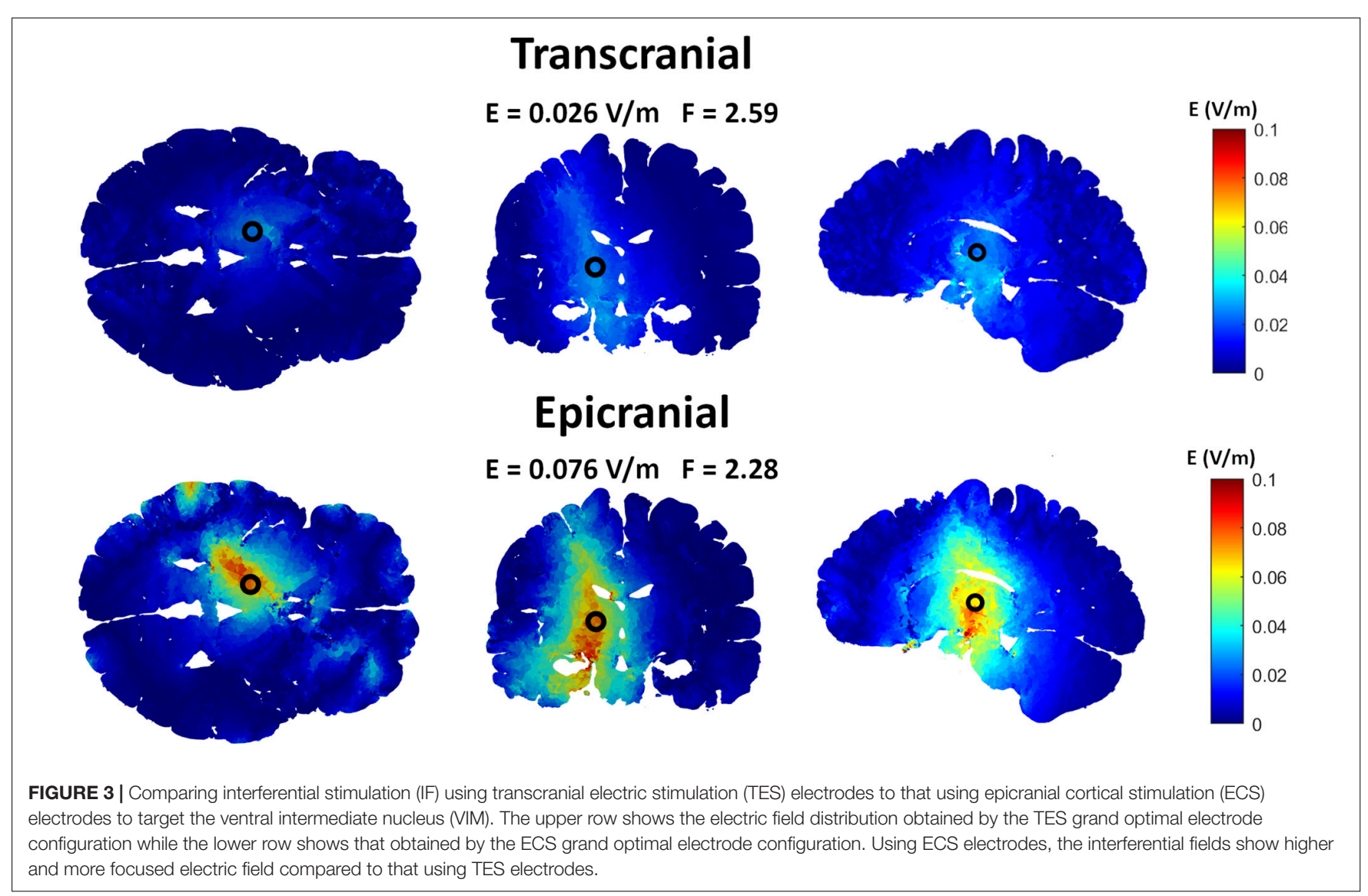


TABLE 1 | Comparing the results of interferential stimulation (IF) using epicranial cortical stimulation (ECS) to that using transcranial electric stimulation (TES).

\begin{tabular}{lcccc}
\hline Target & $\begin{array}{c}\text { Epicranial } \\
\text { EField (V/m) }\end{array}$ & $\begin{array}{c}\text { Transcranial } \\
\text { EField (V/m) }\end{array}$ & $\begin{array}{c}\text { Epicranial } \\
\text { focality (cm) }\end{array}$ & $\begin{array}{c}\text { Transcranial } \\
\text { focality (cm) }\end{array}$ \\
\hline STN & 0.064 & 0.009 & 1.90 & 2.02 \\
VIM & 0.076 & 0.026 & 2.28 & 2.59 \\
Hippocampus & 0.037 & 0.019 & 2.10 & 2.35 \\
Insula & 0.064 & 0.008 & 1.86 & 1.94 \\
\hline
\end{tabular}

TABLE 2 | The effect of decreasing the number of electrodes on the electric field strength and focality.

\begin{tabular}{lccccc}
\hline Target & $\begin{array}{c}\text { All } \\
\text { electrodes } \\
\text { electric } \\
\text { field } \mathbf{( V / m )}\end{array}$ & $\begin{array}{c}\text { Limited } \\
\text { electrodes } \\
\text { electric } \\
\text { field } \mathbf{( V / m )}\end{array}$ & $\begin{array}{c}\text { All } \\
\text { electrodes } \\
\text { focality } \\
\mathbf{( c m )}\end{array}$ & $\begin{array}{c}\text { Limited } \\
\text { electrodes } \\
\text { focality } \\
\mathbf{( c m )}\end{array}$ & $\begin{array}{c}\text { Number of } \\
\text { electrodes }\end{array}$ \\
\hline STN & 0.064 & 0.10 & 1.90 & 2.68 & 6 \\
VIM & 0.076 & 0.08 & 2.28 & 3.18 & 7 \\
Hippocampus & 0.037 & 0.044 & 2.10 & 2.88 & 7 \\
Insula & 0.064 & 0.065 & 1.86 & 2.49 & 7 \\
\hline
\end{tabular}

(i.e., $1 \mathrm{~mA}$ peak amplitude and $2 \mathrm{~mA}$ peak to peak). This solution was selected as the grand optimal configuration and was used for further analysis.

\section{Interferential Stimulation-Epicranial Cortical Stimulation vs. Interferential Stimulation-Transcranial Electric Stimulation}

Epicranial cortical stimulation delivers stronger and more focused non-interferential electric field than TES (Khatoun et al., 2019). To test if this also applies to interferential fields, we compared the results obtained from IF-ECS to that of IF-TES. Figure 3 shows an example of the results obtained for each of the two techniques with the VIM being the target. The results show that IF-ECS produces more focused $(2.28 \mathrm{~cm}$ vs. $2.59 \mathrm{~cm})$ and stronger interferential fields $(0.076 \mathrm{~V} / \mathrm{m}$ vs. $0.026 \mathrm{~V} / \mathrm{m})$ than IF-TES, respectively. These results were similar for all selected targets (see Table 1). When the same stimulation amplitude ( $1 \mathrm{~mA}$, baseline to peak) is used for the same target, IF-ECS shows an average of 3.8 times stronger and 9\% more focused fields.

\section{Limiting the Number of Epicranial Electrodes}

Figure 4 is an example comparing the results obtained using 19 electrodes to that using the major electrodes counting for at least $75 \%$ of the applied current (7 electrodes in this example). The results show a less focused and more spread electric field when using a smaller number of electrodes. On the other hand,

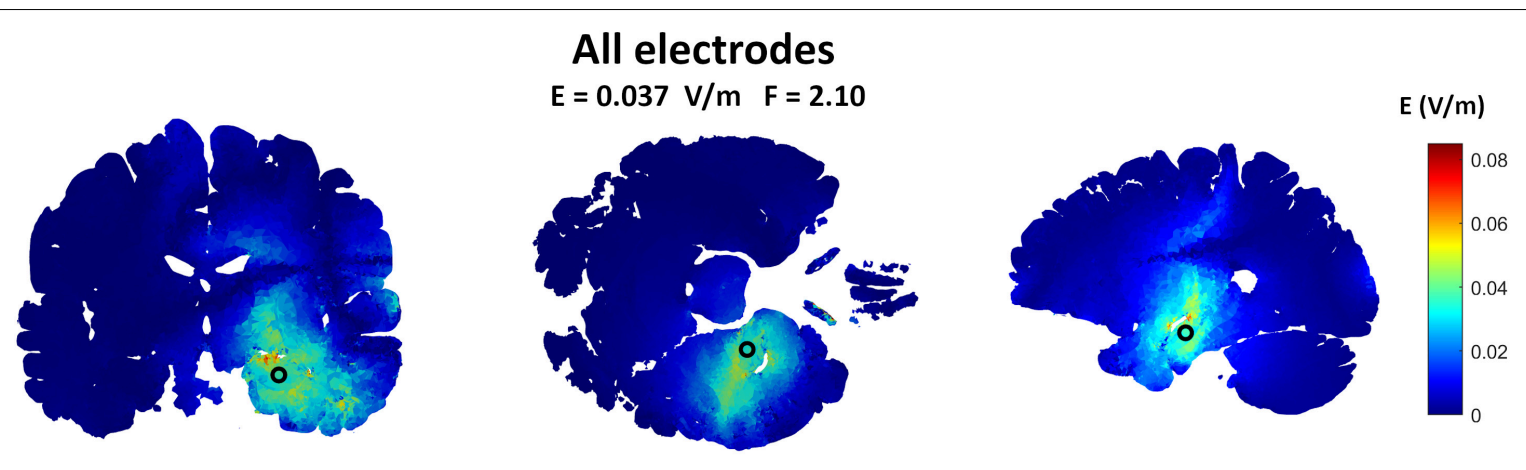

\section{Limited number of electrodes \\ $E=0.044 \mathrm{~V} / \mathrm{m} \quad F=\mathbf{2 . 8 8}$}
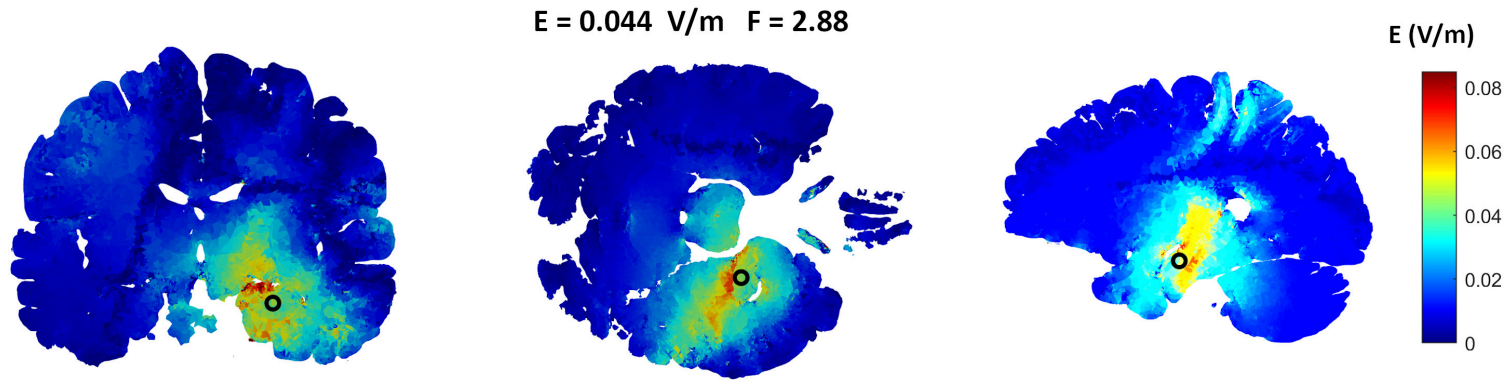

FIGURE 4 | The effects of limiting the number of implanted ECS electrodes on the induced interferential field to target the hippocampus. The upper row shows the electric field distribution obtained by the ECS grand optimal electrode configuration when all the electrodes were used while the lower row shows the fields obtained when only the most essential electrodes were selected. The most essential electrodes were defined as the electrodes contributing to at least $75 \%$ of the injected current for both sources. This was a total of seven electrodes in this example. As shown, the fields are a bit less focused when the number of electrodes were decreased. But by readjusting the total amount of injected current to reach the maximum allowed values (1 mA peak amplitude), the electric field amplitude at the target was higher. This is in line with the amplitude-focality trade-off already discussed in this paper. 
the electric field strength is increased. Table 2 summarizes the results for all selected targets. The number of selected electrodes counting for at least $75 \%$ of the total current varied between 6 and 7 electrodes. On average, this resulted in a $28 \%$ less focused but $346 \%$ stronger fields.

\section{DISCUSSION AND CONCLUSION}

The first aim of this study was to use computational modeling to optimize the electrodes configuration and test the ability of IFECS to target subcortical regions. The optimization method we applied was already investigated in the context of TES and it was shown to successively work (Huang et al., 2020). In this study, the results from the applied optimization technique show that using IF-ECS it is also feasible to target subcortical brain regions while avoiding other brain areas. There was a tradeoff between the electric field focality and amplitudes. When more electric field was permitted in non-target regions, by setting higher $\mathrm{P}_{\max }$ values, higher electric fields were reached in the target regions, but this comes at the cost of focality. This is in line with the findings using transcranial electrodes (Huang et al., 2020).

The second aim was to compare the outcomes of IF-ECS and IF-TES. It has already been shown that ECS induces a stronger and more focused cortical electric fields than TES. However, this is the first study to compare the interferential fields induced by both techniques. The results show that IF-ECS induces higher and more focused interferential fields than IF-TES. On average this was an increase of 3.8 times in field amplitude and an improve of $9 \%$ in focality. Moreover, ECS has the advantage that higher stimulation amplitude are achievable, due to the insulating back layer that blocks the current from passing through the skin (Khatoun et al., 2019). Therefore, a much stronger IF-ECS can be induced by increasing the stimulation amplitude above typical TES values. However, the advantages of IF-ECS come at the cost of invasiveness. IF-ECS would require a surgical intervention to have electrodes placed subcutaneously under the skin and directly over the skull. Thus, limiting the number of implanted electrodes becomes important. Our study shows that limiting the number of electrodes comes at the cost of electric field focality.

A limitation of this study is that it focuses on the induced interferential fields in the brain but not the non-inferential ones. However, this is based on our assumption that neurons will respond to low frequency electric fields but not to that with high frequencies. While there is evidence to support our assumption

\section{REFERENCES}

Akhtari, M., Bryant, H. C., Mamelak, A. N., Flynn, E. R., Heller, L., Shih, J. J., et al. (2002). Conductivities of three-layer live human skull. Brain Topogr. 14, 151-167. doi: 10.1023/a:1014590923185

Alekseichuk, I., Mantell, K., Shirinpour, S., and Opitz, A. (2019). Comparative modeling of transcranial magnetic and electric stimulation in mouse, monkey, and human. Neuroimage 194, 136-148. doi: 10.1016/j.neuroimage.2019.03.044

Antal, A., Alekseichuk, I., Bikson, M., Brockmöller, J., Brunoni, A. R., Chen, R., et al. (2017). Low intensity transcranial electric stimulation: safety, ethical, legal regulatory and application guidelines. Clin. Neurophysiol. 128, 1774-1809. doi: 10.1016/j.clinph.2017.06.001
(Hutcheon and Yarom, 2000; Grossman et al., 2017), testing it is beyond the scope of this study.

Various studies have focused on the effects of anatomical differences and electrode dimensions on the electric field distribution during transcranial and epicranial stimulation (Laakso et al., 2015; Khatoun et al., 2019). A recent study investigated the effect of anatomical differences on the distribution of the interferential fields (Lee et al., 2020). Their results show that different subjects have different optimal montages to target the same brain region. Furthermore, the electric field values at the target varied between the subjects, but all subjects showed relatively stronger fields in the target compared to that in non-target regions. We believe that similar effects on the results would be obtained using epicranial electrodes. However, we did not investigate that in our study.

Epicranial cortical stimulation is a novel technique that was shown to induce strong and focused electric field in the cortex (Khatoun et al., 2019). This study shows that combining ECS with IF is a promising approach for delivering subcortical stimulation where no craniotomy is required. ECS is more invasive that TES but has the potential to deliver stronger and more focused electric fields in the brain. Thus, ECS and ECS combined with IF have the potential to fill a niche in the neuromodulation field for a number of clinical applications.

\section{DATA AVAILABILITY STATEMENT}

The raw data supporting the conclusions of this article will be made available by the authors, without undue reservation.

\section{AUTHOR CONTRIBUTIONS}

AK and ML: conceptualization, methodology, writing, and review. BA: methodology, writing, and review. All authors contributed to the article and approved the submitted version.

\section{FUNDING}

This work was supported by the Fonds Wetenschappelijk Onderzoek (FWO), project number G0B4520N. AK is a junior postdoc fellow at FWO, project number $1217920 \mathrm{~N}$. BA is SB Ph.D. fellow at FWO.
Bestmann, S., and Walsh, V. (2017). Transcranial electrical stimulation. Curr. Biol. 27, R1258-R1262.

Bikson, M., Grossman, P., Thomas, C., Zannou, A. L., Jiang, J., Adnan, T., et al. (2016). Safety of transcranial direct current stimulation: evidence based update 2016. Brain Stimul. 9, 641-661.

Brittain, J.-S., and Cagnan, H. (2018). Recent trends in the use of electrical neuromodulation in Parkinson's disease. Curr. Behav. Neurosci. Rep. 5, 170178. doi: 10.1007/s40473-018-0154-9

Datta, A., Bansal, V., Diaz, J., Patel, J., Reato, D., and Bikson, M. (2009). Gyriprecise head model of transcranial direct current stimulation: improved spatial focality using a ring electrode versus conventional rectangular pad. Brain Stimul. 2, 201-207.e1. doi: 10.1016/j.brs.2009.03.005 
Deng, Z.-D., Lisanby, S. H., and Peterchev, A. V. (2013). Electric field depthfocality tradeoff in transcranial magnetic stimulation: simulation comparison of 50 coil designs. Brain Stimul. 6, 1-13.

EASEE (2021). Epilepsy - Developing the EASEE System-Precisis AG. Available online at: https://precisis.de/en/experience/clinical-studies (accessed September 18, 2021).

Fenoy, A. J., and Simpson, R. K. J. (2014). Risks of common complications in deep brain stimulation surgery: management and avoidance. J. Neurosurg. 120, 132-139. doi: 10.3171/2013.10.JNS131225

Fernández-Corazza, M., Turovets, S., and Muravchik, C. H. (2020). Unification of optimal targeting methods in transcranial electrical stimulation. Neuroimage 209:116403. doi: 10.1016/j.neuroimage.2019.116403

Gabriel, C., Peyman, A., and Grant, E. H. (2009). Electrical conductivity of tissue at frequencies below $1 \mathrm{MHz}$. Phys. Med. Biol. 54, 4863-4878. doi: 10.1088/00319155/54/16/002

Giacometti, P., Perdue, K. L., and Diamond, S. G. (2014). Algorithm to find high density EEG scalp coordinates and analysis of their correspondence to structural and functional regions of the brain. J. Neurosci. Methods 229, 84-96. doi: 10.1016/j.jneumeth.2014.04.020

Grossman, N., Bono, D., Dedic, N., Kodandaramaiah, S. B., Rudenko, A., Suk, H. J., et al. (2017). Noninvasive deep brain stimulation via temporally interfering electric fields. Cell 169, 1029-1041.e16. doi: 10.1016/j.cell.2017.05.024

Guo, L., Guvanasen, G. S., Liu, X., Tuthill, C., Nichols, T. R., and DeWeerth, S. P. (2013). A PDMS-based integrated stretchable microelectrode array (isMEA) for neural and muscular surface interfacing. IEEE Trans. Biomed. Circuits Syst. 7, 1-10. doi: 10.1109/TBCAS.2012.2192932

Huang, Y., Datta, A., and Parra, L. C. (2020). Optimization of interferential stimulation of the human brain with electrode arrays. J. Neural Eng. 17, 36023. doi: 10.1088/1741-2552/ab92b3

Huang, Y., Liu, A. A., Lafon, B., Friedman, D., Dayan, M., Wang, X., et al. (2017). Measurements and models of electric fields in the in vivo human brain during transcranial electric stimulation. Elife 6:e18834.

Hutcheon, B., and Yarom, Y. (2000). Resonance, oscillation and the intrinsic frequency preferences of neurons. Trends Neurosci. 23, 216-222. doi: 10.1016/ s0166-2236(00)01547-2

Iacono, M. I., Neufeld, E., Akinnagbe, E., Bower, K., Wolf, J., Vogiatzis Oikonomidis, I., et al. (2015). IDA: a multimodal imaging-based detailed anatomical model of the human head and neck. PLoS One 10:e124126. doi: 10.1371/journal.pone.0124126

Khatoun, A., Asamoah, B., and Mc Laughlin, M. (2019). Investigating the feasibility of epicranial cortical stimulation using concentric-ring electrodes: a novel minimally invasive neuromodulation method. Front. Neurosci. 13:773. doi: 10. 3389/fnins.2019.00773

Khatoun, A., Breukers, J., Op de Beeck, S., Nica, I. G., Aerts, J. M., Seynaeve, L., et al. (2018). Using high-amplitude and focused transcranial alternating current stimulation to entrain physiological tremor. Sci. Rep. 8:4927.

Laakso, I., Tanaka, S., Koyama, S., De Santis, V., and Hirata, A. (2015). Inter-subject variability in electric fields of motor cortical tDCS. Brain Stimul. 8, 906-913. doi: 10.1016/j.brs.2015.05.002

Lafon, B., Henin, S., Huang, Y., Friedman, D., Melloni, L., Thesen, T., et al. (2017). Low frequency transcranial electrical stimulation does not entrain sleep rhythms measured by human intracranial recordings. Nat. Commun. 8:1199.

Lee, D. J., Lozano, C. S., Dallapiazza, R. F., and Lozano, A. M. (2019). Current and future directions of deep brain stimulation for neurological and psychiatric disorders: JNSPG 75th anniversary invited review article. J. Neurosurg. JNS 131, 333-342. doi: 10.3171/2019.4.jns181761

Lee, S., Lee, C., Park, J., and Im, C.-H. (2020). Individually customized transcranial temporal interference stimulation for focused modulation of deep brain structures: a simulation study with different head models. Sci. Rep. 10:11730. doi: 10.1038/s41598-020-68660-5

Lewis, P. M., Thomson, R. H., Rosenfeld, J. V., and Fitzgerald, P. B. (2016). Brain neuromodulation techniques: a review. Neuroscientist 22, 406-421. doi: $10.1177 / 1073858416646707$

Lozano, A. M., Lipsman, N., Bergman, H., Brown, P., Chabardes, S., Chang, J. W., et al. (2019). Deep brain stimulation: current challenges and future directions. Nat. Rev. Neurol. 15, 148-160. doi: 10.1038/s41582-018-0128-2

Mark, J. E. (1999). Polymer Data Handbook. Oxford: Oxford University Press.

Meacham, K. W., Giuly, R. J., Guo, L., Hochman, S., and DeWeerth, S. P. (2008). A lithographically-patterned, elastic multi-electrode array for surface stimulation of the spinal cord. Biomed. Microdevices 10, 259-269. doi: 10.1007/s10544-0079132-9

Moreno-Duarte, I., Gebodh, N., Schestatsky, P., Guleyupoglu, B., Reato, D., Bikson, M., et al. (2014). "Chapter 2-Transcranial electrical stimulation: transcranial direct current stimulation (tDCS), transcranial alternating current stimulation (tACS), transcranial pulsed current stimulation (tPCS), and transcranial random noise stimulation (tRNS)," in The Stimulated Brain, ed. R. Cohen Kadosh (London: Academic Press), 35-59. doi: 10.1016/B978-0-12-404704-4. 00002-8

Ochoa, M., Wei, P., Wolley, A. J., Otto, K. J., and Ziaie, B. (2013). A hybrid PDMSparylene subdural multi-electrode array. Biomed. Microdevices 15, 437-443. doi: 10.1007/s10544-013-9743-2

Opitz, A., Falchier, A., Yan, C. G., Yeagle, E. M., Linn, G. S., Megevand, P., et al. (2016). Spatiotemporal structure of intracranial electric fields induced by transcranial electric stimulation in humans and nonhuman primates. Sci. Rep. 6:31236. doi: 10.1038/srep31236

Peters, M. J., Stinstra, G., and Hendriks, M. (2001). Estimation of the electrical conductivity of human tissue. Electromagnetics 21, 545-557.

Vöröslakos, M., Takeuchi, Y., Takeuchi, K., Zombori, T., Oliva, A., FernándezRuiz, A., et al. (2018). Direct effects of transcranial electric stimulation on brain circuits in rats and humans. Nat. Commun. 9:483. doi: 10.1038/s41467-01802928-3

Conflict of Interest: The authors declare that the research was conducted in the absence of any commercial or financial relationships that could be construed as a potential conflict of interest.

Publisher's Note: All claims expressed in this article are solely those of the authors and do not necessarily represent those of their affiliated organizations, or those of the publisher, the editors and the reviewers. Any product that may be evaluated in this article, or claim that may be made by its manufacturer, is not guaranteed or endorsed by the publisher.

Copyright (C) 2021 Khatoun, Asamoah and Mc Laughlin. This is an open-access article distributed under the terms of the Creative Commons Attribution License (CC BY). The use, distribution or reproduction in other forums is permitted, provided the original author(s) and the copyright owner(s) are credited and that the original publication in this journal is cited, in accordance with accepted academic practice. No use, distribution or reproduction is permitted which does not comply with these terms. 